NUCLEAR INSTRUMENTS AND METHODS II6 (1974) 105-107; C NORTH-HOLLAND PUBLISHING CO.

\title{
THE ANALYSIS OF GAMMA-GAMMA DIRECTIONAL CORRELATIONS IN A TRIPLE CASCADE*
}

\author{
G. Y. CHOW \\ Physics Department, Saginaw Valley College, University Center, Michigan, U.S.A. \\ P. L. GARDULSKI and M. L. WIEDENBECK \\ Physics Department, University of Michigan, Ann Arbor, Michigan 48104, U.S.A.
}

Received 21 August 1973

If a triple cascade exists among the nuclear levels of an isotope, the (1-3) directional correlation coefficients combined with those of the (1-2) and (2-3) cascades offer an effective way for determining mixing ratios or spin sequences. A formula is given to

\section{Introduction}

It has been pointed out ${ }^{1}$ ) that gamma-gamma directional correlations for a (1-3) cascade with the intermediate transition unobserved can be used to obtain multipolarities of transitions and spins of nuclear energy levels. Measurements on such a cascade demand good resolution of the detectors and the analysis involves spins of upper, lower and intermediate levels as well as the multipolarities of the first, third and the unobserved transitions. The advent of $\mathrm{Ge}(\mathrm{Li})$ detectors has provided adequate resolution for these measurements. Taylor et al. ${ }^{2}$ ) issued a set of tables for first-third correlations while Ramayya et $a^{3}{ }^{3}$ ) generated graphs for several spins and multipolarities. The usefulness of these tables and graphs is limited by the inherent assumption of pure multipolarities in two of the three transitions. Even in the case of consecutive cascades, tables ${ }^{2}$ ) and graphs $^{4,5}$ ) are constructed in such a way that the user must repeatedly choose a series of particular values for the other mixing ratio in order to determine the one in question or to select a proper spin sequence for the cascide. This is especially cumbersome when the $A_{44}$ direstional correlation coefficients have large uncertainties. Another common practice in determining mixing ratios of transitions is to begin by assuming a transition with higher multipolarity to be pure. Such an assumption is often unjustified. It has been pointed out $^{6}$ ) that this assumption can be eliminated if one measures correlations in (1-3) cascades in addition to those in consecutive ones. In the present work, the

- Work supported in part by a grant from the National Science Foundation. determine the mixing ratios for the intermediate transition directly, regardless of whether or not the other two transitions are mixed. Graphs are introduced for analyzing triple cascades.

reiteration method in ref. 6 is simplified by decoupling the mixing ratio of the unobserved transition from those of the first and third transitions. Graphs similar to those generated by Coleman ${ }^{4}$ ) and Arns and Wiedenbeck $^{5}$ ) for consecutive cascades will be introduced for triple cascades. Since only one mixing ratio is involved, this representation can be used directly to analyze correlation data even when the multipolarities of the other transitions are mixed.

\section{Decoupling of the intermediate transition}

Our mixing ratios follow the sign convention of Dzhelepov et al. ${ }^{7}$ ) who suggested the sign of $\delta$, given by the formulas of Biedenharn and $\operatorname{Rose}^{8}$ ), be reversed if the transition is the second in the cascade and otherwise left unchanged. Thus, the sign convention adopted here is opposite to that of Krane and Steffen ${ }^{9}$ ). Our notation follows that of ref. 10. The scheme is workable when a triple cascade as shown in fig. 1 exists among the levels of the isotope and the $\gamma_{1}-\gamma_{2}, \gamma_{2}-\gamma_{3}$ and $\gamma_{1}-\gamma_{3}$ directional correlations can all be determined. For $\gamma_{1}-\gamma_{2}$ cascade, one has

$$
A_{k k}\left(\gamma_{1} \gamma_{2}\right)=A_{k}\left(\gamma_{1}\right) A_{k}\left(\gamma_{2}\right) \text {. }
$$

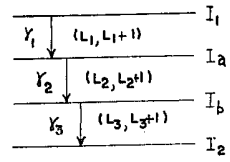

Fig. 1. Gamma transitions in a triple cascade. 
106

For the $\gamma_{2}-\gamma_{3}$ cascade, one has

$$
A_{k k}\left(\gamma_{2} \gamma_{3}\right)=A_{k}^{\prime}\left(\gamma_{2}\right) A_{k}\left(\gamma_{3}\right)
$$

For the $\gamma_{1}-\gamma_{3}$ cascade, one has

$$
A_{k k}\left(\gamma_{1} \gamma_{3}\right)=A_{k}\left(\gamma_{1}\right) U_{k k}\left(\gamma_{2}\right) A_{k}\left(\gamma_{3}\right)
$$

The intermediate transition can be decoupled from the first and third transitions by multiplying eqs. (1) and (2) and then dividing by eq. (3),

$$
R_{k}=\frac{A_{k}\left(\gamma_{2}\right) A_{k}^{\prime}\left(\gamma_{2}\right)}{U_{k k}\left(\gamma_{2}\right)}
$$

where

$$
R_{k}=\frac{A_{k k}\left(\gamma_{1} \gamma_{2}\right) A_{k k}\left(\gamma_{2} \gamma_{3}\right)}{A_{k k}\left(\gamma_{1} \gamma_{3}\right)}
$$

If the expressions for $A_{k}\left(\gamma_{2}\right), A_{k}^{\prime}\left(\gamma_{2}\right)$ and $U_{k k}\left(\gamma_{2}\right)$ are substituted into eq. (4), one obtains a fourth-order equation in the mixing ratios of the intermediate transition, $\delta_{2}$,

$$
\begin{aligned}
& \left(c_{k} f_{k}-g_{k} R_{k}\right) \delta_{2}^{4}+2\left(c_{k} e_{k}-b_{k} f_{k}\right) \delta_{2}^{3}+ \\
& +\left(a_{k} f_{k}-4 b_{k} e_{k}+c_{k} d_{k}+h_{k} R_{k}-g_{k} R_{k}\right) \delta_{2}^{2}+ \\
& \quad+2\left(a_{k} e_{k}-b_{k} d_{k}\right) \delta_{2}+\left(a_{k} d_{k}+h_{k} R_{k}\right)=0
\end{aligned}
$$

where

$$
\begin{aligned}
a_{k} & =F_{k}\left(L_{2}, L_{2}, I_{b}, I_{a}\right), \\
b_{k} & =F_{k}\left(L_{2}, L_{2}^{\prime}, I_{b}, I_{a}\right), \\
c_{k} & =F_{k}\left(L_{2}^{\prime}, L_{2}^{\prime}, I_{b}, I_{a}\right), \\
d_{k} & =F_{k}\left(L_{2}, L_{2}, I_{a}, I_{b}\right), \\
e_{k} & =F_{k}\left(L_{2}, L_{2}^{\prime}, I_{a}, I_{b}\right), \\
f_{k} & =F_{k}\left(L_{2}^{\prime}, L_{2}^{\prime}, I_{a}, I_{b}\right), \\
g_{k} & =(-1)^{I_{a}+I_{b}}\left[\left(2 I_{a}+1\right)\left(2 I_{b}+1\right)\right]^{\frac{1}{2}}(-1)^{L_{2}^{\prime}}\left\{\begin{array}{lll}
I_{b} & I_{b} & k \\
I_{a} & I_{a} & L_{2}^{\prime}
\end{array}\right\},
\end{aligned}
$$

and

$$
\left.\begin{array}{rl}
h_{k}=(-1)^{I_{a}+I_{b}}\left[\left(2 I_{a}+1\right)\left(2 I_{b}+1\right)\right]^{\frac{1}{2}} & (-1)^{L_{2}+1} \times \\
& \times\left\{\begin{array}{l}
I_{b}, I_{b} k \\
I_{a} I_{a}
\end{array} L_{2}\right.
\end{array}\right\} .
$$

It is evident from eqs. (6) and (7) that $\delta_{2}$ is not functionally dependent on the quantities associated solely with the first and third transitions, i.e. $L_{1}, L_{1}^{\prime}$, $L_{3}, L_{3}^{\prime}, I_{1}, I_{2}, \delta_{1}$ and $\delta_{3}$, except via the ratios of experimentally deduced correlation coefficients, $\boldsymbol{R}_{k}$.
A maximum of four solutions for $\delta_{2}$ can result from each value of $\boldsymbol{R}_{2}$. However, usually only one value of $\delta_{2}$ is consistent with both the measured values of $R_{2}$ and $R_{4}$. Once $\delta_{2}$ is determined, $\delta_{1}$ and $\delta_{3}$ or the upper and lower level spins can be deduced by conventional methods $^{8}$ ) or from graphical representations ${ }^{4,5}$ ). Eq. (6) can be rearranged to read

$$
\begin{aligned}
R_{k}= & {\left[c_{k} f_{k} \delta_{2}^{4}+2\left(c_{k} e_{k}-b_{k} f_{k}\right) \delta_{2}^{3}+\right.} \\
& +\left(a_{k} f_{k}-4 b_{k} e_{k}+c_{k} d_{k}\right) \delta_{2}^{2}+ \\
& \left.+2\left(a_{k} e_{k}-b_{k} d_{k}\right) \delta_{2}+a_{k} d_{k}\right] \times \\
& \times\left(1+\delta_{2}^{2}\right)^{-1}\left(g_{k} \delta_{2}^{2}-h_{k}\right)^{-1} .
\end{aligned}
$$

Tables and graphs of $R_{k}$ versus $\delta_{2}$ can be constructed by using eq. (8). Also, $R_{2}$ versus $R_{4}$ parametric plots (with $\delta_{2}$ as the parameter) can be generated. Thus $R_{k}$ may turn out to be a very useful quantity in analyzing correlation data in a triple cascade.

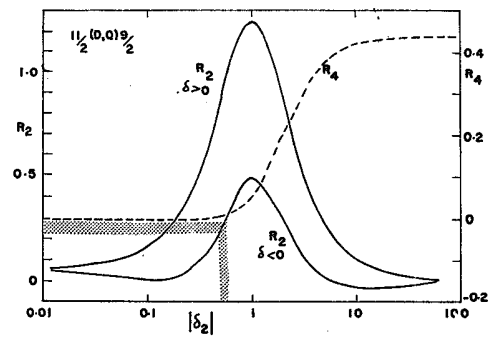

Fig. 2. $R_{2}$ and $R_{4}$ vs $\delta_{2}$ for the $11 / 2(D, Q) 9 / 2$ intermediate transition.

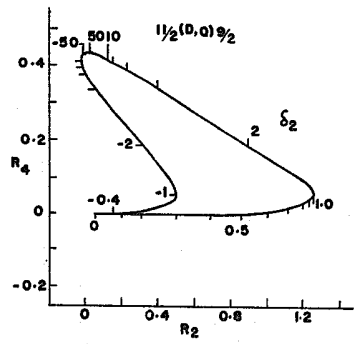

Fig. 3. $R_{4}$ vs $R_{2}$ for the $11 / 2(D, Q) 9 / 2$ intermediate transition. $\delta_{2}$ is listed on the curve. 
It is evident from the symmetry of the expression for $R_{k}$ in eq. (8) that, if the sequence $I_{a}\left(L_{2}, L_{2}^{\prime}\right) I_{b}$ is reversed but the same $R_{k}$ is maintained, $\delta_{2}$ will only change its sign. Therefore, tables and graphs for $I_{a}\left(L_{2}, L_{2}^{\prime}\right) I_{b}$ can be used for the $I_{b}\left(L_{2}, L_{2}^{\prime}\right) I_{a}$ transition provided the sign of $\delta_{2}$ obtained is reversed.

One can classify the graphs into three groups depending on whether the last factor $\left(g_{k} \delta_{2}^{2}-h_{k}\right)$ in eq. (8) can vanish for some values of $\delta_{2}$. For group 1 , $g_{k}$ and $h_{k}$, which are essentially $6 j$ symbols, have opposite signs. Thus, $R_{2}$ and $R_{4}$ should be finite for all $\delta_{2}$. Graphs belonging to this group should have shapes similar to those in example 1 . Graphs in group 2 allow either $R_{2}$ or $R_{4}$ to go to infinity for some $\delta_{2}$. Graphs for group 3 can have $R_{2}$ and $R_{4}$ going to infinity for some values of $\delta_{2}$.

\section{Ilustration, ${ }^{175} \mathrm{Lu}$}

As an example, the triple-cascade $\operatorname{data}^{11}$ ) for the

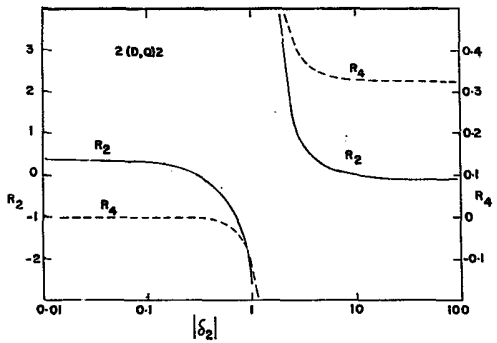

Fig. 4. $R_{2}$ and $R_{4}$ vs $\delta_{2}$ for the $2(D, Q) 2$ intermediate transition.

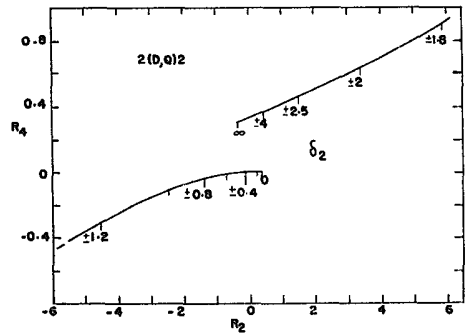

Fig. 5. $R_{4}$ vs $R_{2}$ for the $2(\mathrm{D}, \mathrm{Q}) 2$ intermediate transition. $\delta_{2}$ is listed on the curves.
Table 1

Data used in the determination of mixing ratios of the intermediate transitions in triple cascades for ${ }^{175} \mathrm{Lu}$.

\begin{tabular}{lll}
\hline $\begin{array}{c}\text { Cascade } \\
(E \text { in keV })\end{array}$ & \multicolumn{1}{c}{$A_{22}$} & \\
\hline & $A_{44}$ & \\
\hline$(145-138)$ & $-0.225 \pm 0.004$ & \\
& $-0.003 \pm 0.006$ & \\
$(138-114)$ & $+0.278 \pm 0.008$ & ${ }_{n} R_{2}=0.274 \pm 0.022$ \\
& & \\
& $+0.080 \pm 0.011$ & \\
& & \\
& & Intermediate sequence \\
& & $11 / 2(D, Q) 9 / 2$ \\
$(145-[138]-114)$ & $-0.228 \pm 0.007$ & \\
& $-0.006 \pm 0.009$ & $\delta(138)=-0.540 \pm 0.023$ \\
& &
\end{tabular}

(145-138-114) correlation in ${ }^{175} \mathrm{Lu}$ are listed in table 1. The mixing ratio for the $11 / 2(D, Q) 9 / 2$ transition can be calculated from eq. (6) or read from figs. 2 or 3. While the uncertainty in $R_{4}$ is large, the values of $\boldsymbol{R}_{2}$ and $R_{4}$ as shown in figs. 2 and 3 favor $\delta(138)$ to be $-0.540 \pm 0.023$ which agrees well with the value $-0.55 \pm 0.10$ determined from nuclear orientation ${ }^{12}$ ). Figs. 4 and 5 show the corresponding curves for 2(D, Q) 2 transitions.

All of the commonly encountered sequences up to 15/2 have been plotted and will be supplied upon request.

\section{References}

1) G. B. Arfken, L. C. Biedenharn and M. E. Rose, Phys. Rev. 86 (1952) 761.

2) H. W. Taylor et al., Nuclear Data Tables 9A (1971) 1.

3) A. V. Ramayya et al., Oak Ridge National Laboratory Report ORNL-4619.

4) C. F. Coleman, Nucl. Phys. 5 (1958) 495.

5) R. G. Arns and M. L. Wiedenbeck, Un. of Michigan Technical Report 2375-3-T (1958).

6) G. 'Y. Chow and M. L. Wiedenbeck, accepted for publication in Nucl. Instr. and Meth.

7) B. S. Dzhelepov, M. A. Listengarten and S. A. Shestopalova, Izv. Akad. Nauk. SSSR, Ser. Fiz. 31 (1967) 581.

8) L. C. Biedenharn and M. E. Rose, Rev. Mod. Phys. 25 (1953) 729.

9) K. S. Krane and R. M. Steffen, Phys. Rev. C2 (1970) 724.

10) H. Frauenfelder and R. M. Steffen, Alpha-, beta- and gammaray spectroscopy (Ed. K. Siegbahn, No: th Holland Publ. Co., Amsterdam, 1968), Chapter XIX.

11) G. Y. Chow, P. L. Gardulski and M. L. Wiedenbeck, submitted for publication.

12) K. S. Krane, C. E. Olsen and W. A. Steyert, Nucl. Phys. A197 (1972) 352. 